Journal of Bangladesh Chemical Society, Vol. 25(2), 180-185, 2012

\title{
METHOD DEVELOPMENT AND VALIDATION FOR ESTIMATION OF COMMERCIALLY PRODUCED SEX PHEROMONES IN LURE
}

\author{
SHANKAR MANDAL, A M H RAHMAN, M I R MAMUN, MOHAMMAD SHOEB AND \\ NILUFAR NAHAR* \\ Department of Chemistry, University of Dhaka, Dhaka-1000, Bangladesh
}

\begin{abstract}
A method was developed and validated for quantitation of methyl eugenol and cuelure, the active ingredients of two formulated sex pheromones (bio-pesticides) Mukti and Jhilik by gas chromatography. Technical grade methyl eugenol and cuelure (purity 9596\%) were used as standards. Calibration curves of methyl eugenol and cuelure were linear with correlation coefficient $\left(r^{2}\right) 0.9966$ \& 0.9988 , respectively. Ten replicate analyses were done for both methyl eugenol \& cuelure and quantity of the pheromones in the two formulated products were found to be $0.55 \pm 0.11$ and $0.56 \pm 0.09$ g/lure (Mean \pm $\mathrm{SD})$, respectively. The stability of both pheromones in solution and their diffusion from lures under UV-Vis light was determined for a period of 21 days. The results showed that diffusion of the both pheromones from lure were very fast and about two-third diffused within 10 days after exposure. Methyl eugenol was quite stable in solution, whereas cuelure was found to degrade slowly, and about $15 \%$ cuelure degraded within 21 days. The recovery of methyl eugenol and cuelure from matrix were found to be 80.69 \pm 3.14 and $78.48 \pm 4.32 \%$ (mean \pm SD), respectively, where limit of detection (LOD) \& limit of quantification (LOQ) were found to be $97 \& 80 \mathrm{ppm}$, and $290 \& 240 \mathrm{ppm}$, respectively. The developed method is very simple and can be used for estimation of the two sex pheromones.
\end{abstract}

\section{Introduction}

Sex pheromones are small molecular volatile chemical compounds of plant origin, highly specific to the target, and used to control pests by indirect method. The compounds are non-toxic and have no threat to human health and environment, easy to use and key components of integrated pest management (IPM) programs ${ }^{1,3,4,5}$. In mid 2002, Environmental Protection Agency (EPA), USA gave registration of 36 pheromones, including methyl eugenol (4-allyl-1,2-dimethoxybezene-carboxylate) and cuelure [4-( $p$ acetoxyphenyl)-2-butanone $]^{3,6,7}$. Methyl eugenol and cuelure traps are used for suppression of male oriental fruit fly, Bactrocera dorsalis (Hendel) and melon fly, Bactrocera cucurbitae (Coquillett) ${ }^{3,8}$. In Bangladesh, local manufacturers are now producing methyl eugenol (Mukti) and cuelure (Jhilik) in lure formulation to control fruits and vegetables fly and other insects. In order to implement these environmentalfriendly pest control agents effectively, it is important to measure the amount of volatile active ingredients in the formulated products which are insect attractants ${ }^{3}$. Therefore, an approach was taken to develop a suitable method for determination of active ingredients in the lures and to validate the developed method.

* Author for correspondence; e-mail: nilufarnahar@yahoo.com 


\section{Experimental}

\section{Sampling}

Two formulated sex pheromones, methyl eugenol (Mukti) and cuelure (Jhilik) lures were collected from the Department of Agricultural Extension (DAE), Dhaka. Lures were made by soaking heavy cotton thread containing plastic clip with formulated methyl eugenol or cuelure and packed in tri-laminated pouch with aluminum foil. Blank lures (without active ingredients) were also collected from DAE to perform recovery experiments.

\section{Instruments}

For Gas chromatography a Shimadzu 2025 GC fitted with flame ionization detector (FID) and quartz capillary column (HP-5; $30 \mathrm{~m} \times 0.25 \mathrm{~mm} \times 0.25 \mu \mathrm{m}$ ) were used. The injector and detector temperatures were set at $225^{\circ} \mathrm{C}$ and $275^{\circ} \mathrm{C}$, respectively and nitrogen was used as carrier gas. Separations were performed at $110^{\circ} \mathrm{C}(1 \mathrm{~min}$. hold $) 15^{\circ} \mathrm{C} / \mathrm{min}$. to 220 ${ }^{\circ} \mathrm{C}(6 \mathrm{~min}$. hold $)$ for methyl eugenol and $120{ }^{\circ} \mathrm{C}\left(1 \mathrm{~min}\right.$. hold) $15{ }^{\circ} \mathrm{C} / \mathrm{min}$. to $220{ }^{\circ} \mathrm{C}(6$ min. hold) for cuelure.

\section{Extraction and clean up}

Lure was taken in a sealed ground joint graduated test tube. A mixture of n-hexane and acetone $(1: 1,8 \mathrm{~mL})$ was added to the test tube. The content was ultrasonicated for 30 second, rapped with parafilm and kept overnight (about $12 \mathrm{hrs)}$ ) at room temperature. The extract was transferred in another sealed ground joint graduated test tube. The lure was reextracted for 4 hours with another $7 \mathrm{~mL}$ of same solvent mixture. The two extracts were combined together. A part of the extract was filtered through millipore membrane filters ( $0.45 \mu \mathrm{m}$ pore size) and analyzed by GC-FID.

\section{Recovery experiment}

Replicate recovery experiments of methyl eugenol and cuelure were done by spiking the blank matrix i.e lure and spiking level was $200 \mathrm{ppm}$ in each case. The recovery of cuelure and methyl eugenol were found to be $78.48 \pm 4.32$ and $80.69 \pm 3.14 \%$ (mean \pm SD), respectively.

\section{Stability and diffusion study}

The stability and diffusion of both methyl eugenol and cuelure were studied in a period of 21 days under UV-Vis light. Both formulated lures were exposed under UV-Vis light and active ingredients were determined at $0,3,7,15$ and 21 days after exposure. To determine the stability of the pheromones, the extracts of both formulated lures were also exposed under UV-Vis light and active ingredients were determined at 0, 3, 7, 15 and 21 days after exposure. 


\section{Results and Discussion}

Methyl eugenol and cuelure were analyzed by GC-FID. Both methyl eugenol and cuelure were symmetrically eluted and their retention time were 4.48 and 5.85 min, respectively, with retention factors 4.21 and 5.80 , respectively, and the repeatability of the retention times was less than $1 \%$ for 5 injections. The repeatability of the analytical procedure (as relative standard deviation, RSD \%) was estimated analyzing six replicates of blank lures spiked with an intermediate standard solution of methyl eugenol and cuelure, respectively. Method repeatability (RSD \%) for methyl eugenol and cuelure was found to be 3.56 and $5.50 \%$, respectively.

The limits of detection (LOD) and limit of quantification (LOQ) were determined by injecting serially diluted standard solutions from low to high concentration and LOD \& LOQ were calculated considering each of the compound's peak three and ten times higher than the basal level (i.e. noise). LOD and LOQ for methyl eugenol were found to be 97 and $290 \mathrm{ppm}$, respectively, whereas the values were 80 and $240 \mathrm{ppm}$, respectively for cuelure. Analysis was carried out at split mode rather than split/splitless mode.

LOD and LOQ for both the compounds were found to be high at the split mode (1:70). Split gas liner of GC vaporization chamber is large and in that case there is always loss of compound due to continuous split. In split/splitless gas liner vaporized sample directly go to the column and loss of compound is rather less. Less specificity of the compounds might be another reason for high LOD and LOQ. A linear calibration curve was produced for each standard methyl eugenol and cuelure with the correlation coefficient, $\mathrm{r}^{2}=0.9966$ and 0.9988 , respectively (Figure 1 ).

The value of the correlation coefficient obtained for each calibration curve showed that the correlation between relative peak area and concentration was good.

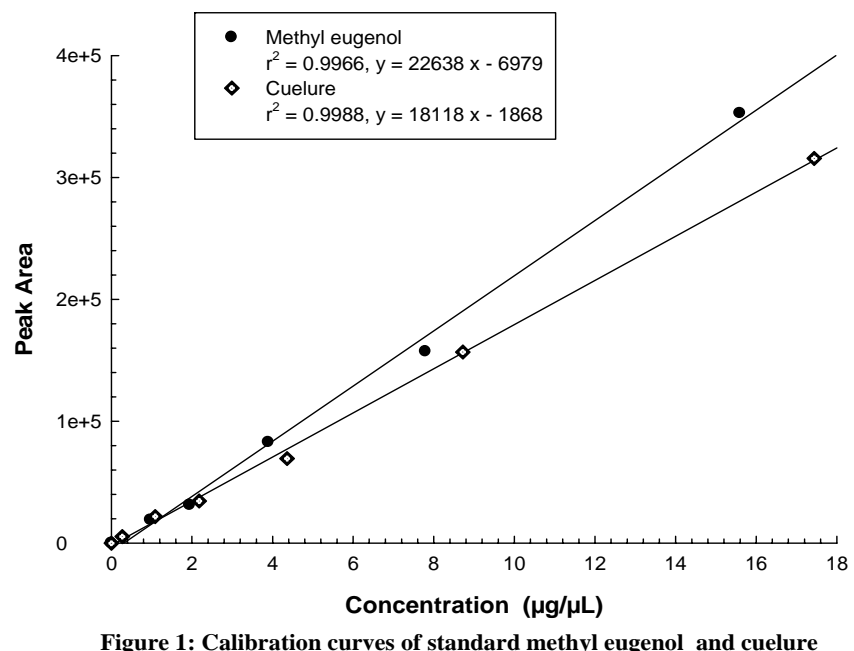

Figure 1: Calibration curves of standard methyl eugenol and cuelure 
Ten replicates analysis were performed for each of the formulated product of cuelure and methyl eugenol to determine amount of active ingredients. Blank lure was used as control. Quantitation of the active ingredients in lures was done using external standard method (Table 1). Two small extra peaks prior to the peak of cuelure in the chromatogram were detected but these were not present either in methyl eugenol or control indicated the presence of impurity or degradation product of the parent compound.

The amount of active ingredients of cuelure and methyl eugenol in lures were found to be $0.56 \pm 0.09$ and $0.55 \pm 0.11$ g/lure (mean \pm SD), respectively. Amount of active ingredient in each of the lure was $0.96 \mathrm{~g}$ according to the manufacturer. As the compounds are highly volatile there is great chance of loss of the compounds during formulation, packaging, extraction \& analysis and because of that about $40 \%$ active ingredients have been lost.

Table 1. Amount of active ingredients of methyl eugenol and cuelure in formulated lures

\begin{tabular}{|c|c|c|c|}
\hline \multicolumn{2}{|c|}{ Cuelure } & \multicolumn{2}{c|}{ Methyl eugenol } \\
\hline Sample ID & Active ingredients (g / lure) & Sample ID & Active ingredients (g / lure)) \\
\hline CL 1 & 0.67 & ML 1 & 0.54 \\
\hline CL 2 & 0.68 & ML 2 & 0.48 \\
\hline CL 3 & 0.49 & ML 3 & 0.75 \\
\hline CL 4 & 0.42 & ML 4 & 0.42 \\
\hline CL 5 & 0.59 & ML 5 & 0.49 \\
\hline CL 6 & 0.61 & ML 6 & 0.52 \\
\hline CL 7 & 0.43 & ML 7 & 0.59 \\
\hline CL 8 & 0.63 & ML 8 & 0.71 \\
\hline CL 9 & 0.56 & ML 9 & 0.41 \\
\hline CL 10 & 0.55 & ML 10 & 0.58 \\
\hline Mean \pm SD & $0.56 \pm 0.09$ & Mean \pm SD & $0.55 \pm 0.11$ \\
\hline
\end{tabular}

Efficacy and effectiveness of sex pheromones depend on its stability and rate of disappearance of active ingredients of formulated lure when applied in agricultural fields. The extract of both formulated lures were exposed under UV-Vis. light and analyzed at 0 , 3, 7, 15 and 21 days after exposure (Figure 2). Methyl eugenol and cuelure were found to decompose under UV-Vis. light, but rate was faster for cuelure than methyl eugenol. About 15\% active ingredients (cuelure) were decomposed at 21 days after exposure, while only trace amounts of methyl eugenol metabolites detected at that period. 


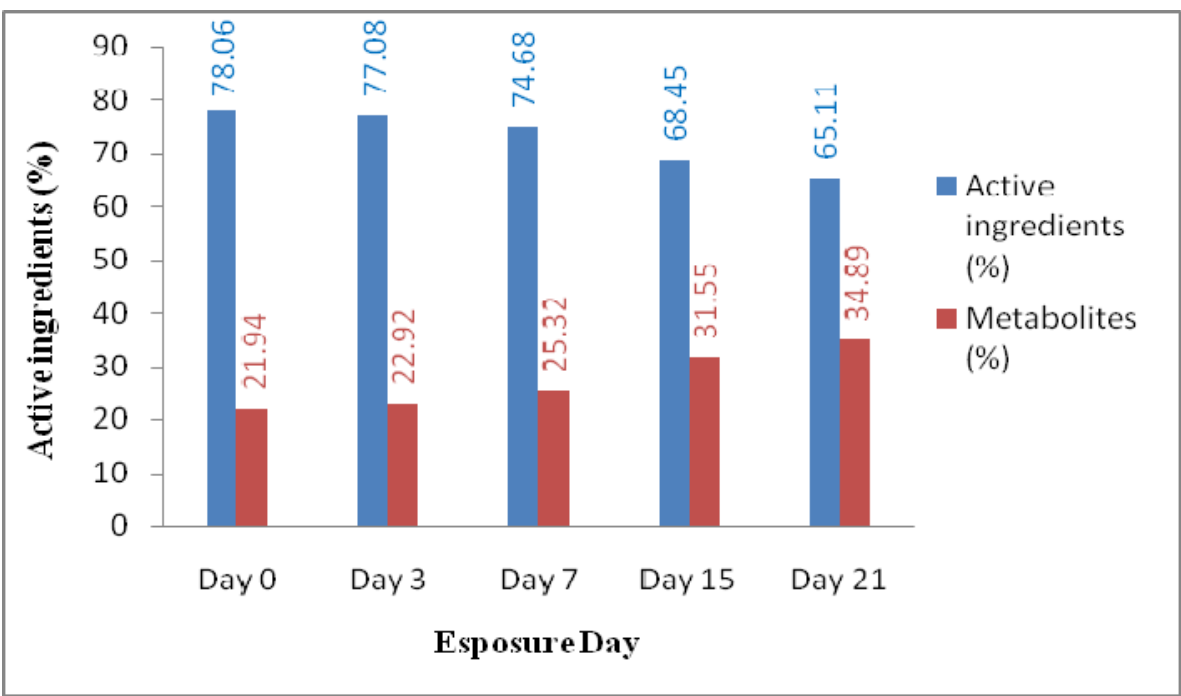

Fig. 2. Stability of cuelure in extract of lure under UV-Vis light

Sex pheromones act via diffusion in IPM program to control pests and its effectiveness depend on diffusion rate. Both methyl eugenol and cuelure are small molecular volatile compounds, so diffuse quickly when exposed in UV-Vis light at room temperature. Results showed (Figure 3) that about two third of both the active ingredients disappeared from lures within ten days of its exposure.

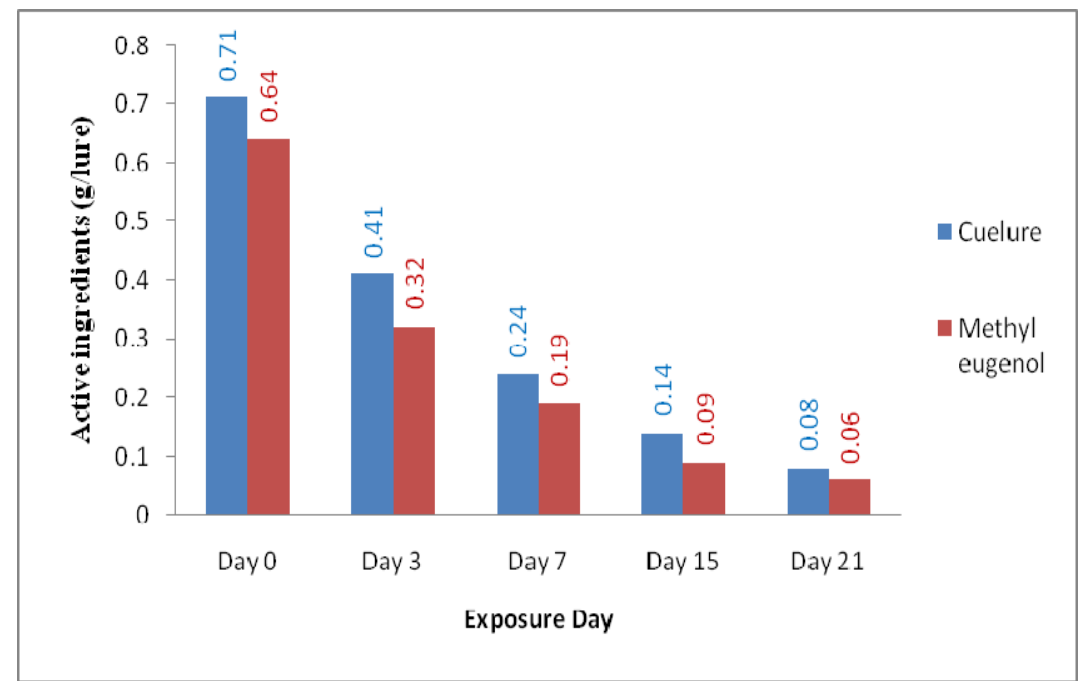

Fig. 3. Disappearence of active ingredients of formulated lures under UV-Vis light. 


\section{Conclusion}

Biopesticides such as sex pheromones can be analyzed by GC-FID very effectively. Both formulated products of cuelure and methyl eugenol have active ingredients to apply in integrated pest management (IPM) program effectively in a period of about 10 days. To reduce the loss of active ingredients attempt should taken for proper lure formulation, storage and packaging, so that sex pheromones will be an effective tool of pest controls system.

\section{Acknowledgement}

The authors are grateful to the International Science Programme (ISP), Uppsala University, Uppsala, Sweden for financial support and Department of Agricultural Extension (DAE), Dhaka to supply standards and samples.

\section{References}

1. U. S. Environmental Protection Agency, “Biopesticides”, May, 2012.

2. H. D. Burges (ed.) "Formulation of Microbial Biopesticides, beneficial microorganisms, nematodes and seed treatments" Kluwer Publishing Academic, Dordrecht, 1998, p 412.

3. U. S. Environmental Protection Agency, "Biopesticides Active ingredients Fact Sheet", May, 2012.

3. B. Steinwand. Personal communication. Washington DC: "US Environmental Protection Agency. Biopesticide” Ombudsman, 2008.

4. H. Kaya \& Lacey (eds.) "Field Manual of Techniques for the Evaluation of Entomopathogens” Kluwer Publishing Academic, Dordrecht, NL, 2000, p 911.

6. G. Ware and D. Whitacre, "An Introduction to Insecticides" 2004. E. Radcliffe, W. Hutchison and Cancelado, R., Radcliffe's "IPM World Textbook". URL: http://ipmworld.umn.edu. St. Paul (MN): University of Minnesota.

7. R. I. Vargas, T. E. Shelly, L. Leblanc and J. C. Piñero. "Recent advances in methyl eugenol and cue-lure technologies for fruit fly detection, monitoring, and control in Hawaii. Vitam Horm”. J Econ Entomol., 2010, 83, 575-95.

8. R. I. Vargas, J. D. Stark, M. H. Kido, H. M. Ketter and L. C. Whitehand. 'Methyl eugenol and cue-lure traps for suppression of male oriental fruit flies and melon flies (Diptera: Tephritidae) in Hawaii: effects of lure mixtures and weathering”. 2000, 93(1), 81.

(Received : 29 July, 2012; Accepted : 05 November, 2012)

Fig. 1. Calibration curves of standard methyl eugenol and cuelure 